\title{
DIFICULDADES RELACIONADAS À IMPLANTAÇÃO E CERTIFICAÇÃO DE SISTEMAS DE GESTÃO DA QUALIDADE EM EMPRESAS CONSTRUTORAS
}

\author{
DIFFICULTIES RELATED TO IMPLEMENTATION AND \\ CERTIFICATION OF QUALITY MANAGEMENT SYSTEMS IN \\ CONSTRUCTION COMPANIES
}

\author{
Marcelo D. Depexe ${ }^{1}$; Edson P. Paladini ${ }^{2}$ \\ ${ }^{1}$ Universidade Federal de Santa Catarina - UFSC - Florianópolis - Brasil marcelodepexe@yahoo.com.br \\ ${ }^{2}$ Universidade Federal de Santa Catarina - UFSC - Florianópolis - Brasil paladini@deps.ufsc.br
}

\begin{abstract}
Resumo
O número de empresas construtoras que buscam uma certificação de seus sistemas de gestão da qualidade tem crescido nos últimos anos, baseados principalmente na ISO 9000 e no PBQP-H. Entretanto, as empresas passam por uma série de dificuldades durante o processo de implantação e certificação do sistema. O presente trabalho tem como objetivo determinar a percepção dessas empresas quanto as principais dificuldades enfrentadas neste processo. A pesquisa é realizada com 14 construtoras atuantes na região da Grande Florianópolis, sob a forma de uma entrevista estruturada, baseada em um questionário, que avalia o grau de importância de cada fator apresentado segundo uma escala de um a cinco (Likert). Dentre os principais resultados, salientam-se os aspectos culturais e a resistência a mudanças, além do excesso de burocracia gerada pelo sistema. Além de colaborar para uma melhor compreensão da evolução da qualidade na construção civil, este artigo procura salientar aspectos críticos para a implantação de sistemas de gestão da qualidade, o que pode auxiliar outras empresas que ainda não completaram ou não iniciaram o processo de certificação.
\end{abstract}

Palavras-chave: qualidade; PBQP-H; dificuldades.

\section{Introdução}

A partir de meados dos anos 90, diversas empresas construtoras têm buscado a implantação e certificação de sistemas de gestão da qualidade. Este movimento teve por base a série de normas ISO 9000 e posteriormente, o PBQP-H (Programa Brasileiro da Qualidade e Produtividade no Habitat). Apesar de todos os benefícios advindos do sistema de gestão da qualidade, diversos autores enumeram vários fatores que dificultam o processo. 
O comprometimento da alta administração é o fator crítico mais citado em pesquisas internacionais, de acordo com Sila e Ebrahimpour (2003). Ao analisar a vivência dos consultores do CTE - Centro de Tecnologia de Edificações junto a 75 empresas construtoras, Souza e Mekbekian (1995) verificam a falta de comprometimento prático com a implementação do programa da qualidade e com sua avaliação, limitando-se a definir a Política da Qualidade. A falta de entendimento do significado e alcance do programa da qualidade é apontada como uma das causas para o desinteresse da alta administração.

Assim como o comprometimento da alta administração é essencial, o comprometimento dos gerentes é muito importante para a sustentação da qualidade. Como a promoção da qualidade em uma empresa é um processo de envolvimento e comprometimento de pessoas, o papel dos gerentes é de suma relevância (TOLOVI JR., 1994). Conforme Liu (1998), embora muitos gerentes possuam experiência em sua área, não possuem os conhecimentos necessários sobre gestão da qualidade. Do seu ponto de vista, não há nada errado no sistema e na gestão atual, o que induz a uma certa relutância quanto ao novo sistema de gestão da qualidade. De acordo com Dalgleish (2004), um dos empecilhos para a melhoria da qualidade é justamente a falsa idéia de que a empresa já trabalha em níveis ótimos e que não há necessidade de melhoria.

Outra dificuldade que as construtoras encontram é a resistência nos níveis hierárquicos mais baixos, o que dificulta seu envolvimento para as atividades voltadas à avaliação e melhoria da qualidade. A adesão dos funcionários às transformações necessárias é ponto fundamental para que os objetivos do programa sejam alcançados (PRADO, AMARAL e TOLEDO, 2001).

A baixa escolaridade dos funcionários da construção civil é também apontada como uma das dificuldades para a execução de obras e a introdução de medidas voltadas para a melhoria da qualidade no setor, conforme Neves (1995). O baixo nível de escolaridade é a principal dificuldade enfrentada por construtoras durante a implantação de sistemas de gestão da qualidade no Piauí, conforme pesquisa realizada por Mendes e Picchi (2005). Resultado semelhante é apresentado por Silveira et al. (2002) no Estado do Rio Grande do Norte.

Observa-se assim que há uma certa resistência a mudanças em todos os níveis hierárquicos, devido a uma cultura organizacional fortemente sedimentada. A construção civil é um setor muito tradicional, no qual as inovações ocorrem de maneira lenta. Os métodos construtivos variam pouco ao longo dos anos, porém, ao se realizar uma padronização de procedimentos, devido à implantação de um sistema de gestão da qualidade, os procedimentos atuais são questionados, com a intenção de determinar a melhor maneira de realizá-los (REIS e MELHADO, 1998). Segundo esses autores, parte da resistência gerada deve-se ao fato de que as pessoas envolvidas diretamente na execução dos serviços não são consultadas para a elaboração de novos procedimentos. Isso gera hostilidade e falta de comprometimento com a proposta de melhoria da qualidade. 
Falhas de comunicação também são apontadas como dificuldades durante a implantação. $\mathrm{O}$ conservadorismo das práticas na indústria da construção civil sugere que existe uma barreira entre departamentos e níveis hierárquicos (LOW e OMAR, 1997). Esses autores afirmam que os departamentos voltam-se para o escopo do seu trabalho, havendo pouca integração de decisões sobre métodos e processos. Como resultado desta falta de comunicação ocorre uma fraca coordenação na organização ou projeto específico.

Além do comprometimento, é necessário que os funcionários tenham a qualificação para executar suas tarefas adequadamente. A importância do treinamento é demonstrada por Antony et al. (2002), ao concluir que treinamento e educação são os fatores críticos de sucesso mais importantes para a gestão da qualidade total. Esta pesquisa foi realizada em Hong Kong, com 32 empresas de diversos segmentos, todas com mais de 250 funcionários e certificadas pela ISO 9000. McIntyre e Kirschenman (2000) apresentam a mesma conclusão ao realizar um levantamento com 151 construtoras nos Estados Unidos da América.

Turk (2006) apresenta uma pesquisa com 68 construtoras na Turquia certificadas pela ISO 9000. Segundo o autor, o aumento da documentação é apontado como a principal desvantagem do sistema de gestão da qualidade. A burocracia do sistema também é a dificuldade mais apontada por um conjunto de aproximadamente 40 empresas construtoras de nove estados brasileiros, de acordo com pesquisa realizada por Bauer e Brandli (2005). O sistema de gestão da qualidade cria sua própria burocracia, com regras e padrões próprios, podendo tornar-se um processo paralelo alheio às atividades operacionais diárias. Se gasta muito tempo reunindo dados irrelevantes e produzindo relatórios inúteis, o que gera grande custos para se manter o sistema (TATIKONDA e TATIKONDA, 1996).

Outros aspectos, como a ansiedade por resultados, a falta de foco no cliente e a falta de liderança também são abordados pela literatura. Uma vez que as empresas necessitam alterar o status quo e desenvolver uma cultura que suporte a gestão da qualidade, os resultados podem demorar a aparecer, de modo que é necessário tempo para se adaptar e aprender a trabalhar com qualidade (LOW e TEO, 2004).

Taylor e Wright (2003) apresentam outra pesquisa realizada no Reino Unido, com 113 organizações. Os autores afirmam que o foco no cliente, juntamente com o comprometimento da alta administração, como os elementos essenciais para o sucesso da implantação de um sistema de gestão da qualidade.

Segundo Sutherland et al. (1995), algumas iniciativas de melhoria da qualidade falham porque as lideranças não conseguem promover a alteração comportamental necessária entre os funcionários, de modo a gerar uma insatisfação com os níveis de qualidade atuais. Deste modo, 
instala-se um desejo de melhoria entre todos os envolvidos. Conforme Lee (1998), a liderança é apontada como um dos elementos mais importantes para a gestão da qualidade.

\section{Método de pesquisa}

O levantamento ocorreu entre os meses de julho e outubro de 2005. Neste período, haviam 38 construtoras certificadas no nível A do PBQP-H no Estado de Santa Catarina. Foram contatadas 21 empresas construtoras das cidades de Florianópolis e São José. Entretanto, sete empresas não demonstraram interesse em colaborar com a pesquisa. Deste modo, o presente levantamento conta com a participação de 14 empresas. Optou-se por pesquisar apenas empresas do nível A porque estas já estão com o processo de implantação completamente concluído, de modo a fornecer uma visão mais ampla e fundamentada a respeito do sistema e do programa.

A pesquisa objetiva determinar a percepção dessas empresas quanto às principais dificuldades enfrentadas durante a implantação e certificação de sistemas de gestão da qualidade. Esta pesquisa é realizada sob a forma de uma entrevista estruturada, baseada em um questionário, com o responsável da qualidade das empresas. O entrevistado expressa sua percepção sobre cada item de acordo com uma escala de um a cinco (Likert).

Todas as questões e os itens que constam no questionário foram baseados em pesquisas similares nacionais e internacionais, notadamente referente à certificação na norma ISO 9000. Uma vez que há compatibilidade entre a ISO 9001:2000 e o PBQP-H, não há problema em se comparar tais pesquisas. Além disso, diversas empresas pesquisadas também possuem a certificação ISO 9001:2000. Em todo momento o entrevistado pôde comentar cada item livremente, o que fornece informações mais substanciais do que a simples informação do nível de percepção, de modo a permitir uma melhor interpretação dos dados obtidos.

$\mathrm{O}$ autor visitou pessoalmente cada empresa para solicitar sua colaboração na pesquisa. Optou-se pela realização de entrevistas para obter-se uma maior quantidade de informações, de modo a proporcionar um melhor entendimento sobre o processo de implantação do sistema de gestão da qualidade e da situação atual do sistema nas empresas. Além disso, a presença do autor

permite o esclarecimento dos itens do questionário, de modo a fornecer maior confiabilidade às respostas.

Os dados obtidos foram analisados com auxílio de uma planilha eletrônica. Foram calculados, para cada item, a média e o desvio-padrão. Realizou-se também uma análise de freqüência das respostas quanto à pontuação na escala de um a cinco. Assim, procedeu-se a análise quantitativa através dos dados obtidos, que podem ser complementados e justificados por uma 
análise de cunho qualitativo, baseada nos comentários realizados pelos entrevistados. A seguir, apresentam-se os resultados obtidos.

\section{Resultados}

Apresentam-se aqui as principais dificuldades enfrentadas pelas 14 empresas pesquisadas durante o processo de implantação do sistema de gestão da qualidade. A Tabela 1 apresenta a média e o desvio-padrão das 14 empresas. Observa-se que as questões relativas à cultura organizacional e o excesso de burocracia gerada pelo sistema representam as maiores dificuldades. A distribuição das pontuações pode ser observada na Tabela 1. A seguir, cada item é analisado separadamente.

Tabela 1 Média, desvio-padrão e distribuição das notas

\begin{tabular}{|c|c|c|c|c|c|c|c|}
\hline \multirow{2}{*}{ Dificuldades durante a implantação } & \multirow{2}{*}{ Média } & \multirow{2}{*}{$\begin{array}{l}\text { Desvio- } \\
\text { padrão }\end{array}$} & \multicolumn{5}{|c|}{ Distribuição das notas } \\
\hline & & & 1 & 2 & 3 & 4 & 5 \\
\hline Cultura organizacional e resistência a mudanças & 4,29 & 0,61 & $0 \%$ & $0 \%$ & $7 \%$ & $57 \%$ & $36 \%$ \\
\hline Burocracia excessiva & 4,14 & 1,10 & $0 \%$ & $14 \%$ & $7 \%$ & $29 \%$ & $50 \%$ \\
\hline Baixo nível de escolaridade dos funcionários & 2,93 & 1,49 & $21 \%$ & $21 \%$ & $21 \%$ & $14 \%$ & $21 \%$ \\
\hline Falta de treinamento & 2,79 & 1,42 & $29 \%$ & $7 \%$ & $36 \%$ & $14 \%$ & $14 \%$ \\
\hline Falta de envolvimento dos funcionários & 2,57 & 1,16 & $21 \%$ & $29 \%$ & $21 \%$ & $29 \%$ & $0 \%$ \\
\hline Falta de participação e conscientização dos colaboradores & 2,50 & 1,22 & $36 \%$ & $7 \%$ & $43 \%$ & $7 \%$ & $7 \%$ \\
\hline Comunicação deficiente & 2,43 & 1,28 & $29 \%$ & $21 \%$ & $29 \%$ & $14 \%$ & $7 \%$ \\
\hline Ansiedade por resultados & 2,36 & 1,15 & $36 \%$ & $7 \%$ & $43 \%$ & $14 \%$ & $0 \%$ \\
\hline Falta de comprometimento da alta administração & 2,36 & 1,50 & $43 \%$ & $14 \%$ & $21 \%$ & $7 \%$ & $14 \%$ \\
\hline Falta de recursos & 1,93 & 1,59 & $71 \%$ & $0 \%$ & $7 \%$ & $7 \%$ & $14 \%$ \\
\hline Falta de liderança & 1,93 & 1,38 & $64 \%$ & $0 \%$ & $21 \%$ & $7 \%$ & $7 \%$ \\
\hline Falta de comprometimento dos gerentes & 1,93 & 1,27 & $50 \%$ & $29 \%$ & $7 \%$ & $7 \%$ & $7 \%$ \\
\hline Falta de foco no cliente & 1,50 & 1,09 & $79 \%$ & $7 \%$ & $0 \%$ & $14 \%$ & $0 \%$ \\
\hline
\end{tabular}

\subsection{Cultura organizacional e resistência a mudanças}

O item cultura organizacional e resistência a mudanças apresenta a maior média de todas as dificuldades vivenciadas pelas empresas durante a implantação do sistema de gestão da qualidade. Observa-se também que este item possui o menor desvio-padrão, o que indica menor variabilidade na opinião das empresas. Isso pode ser visualizado na Tabela 1, que mostra grande concentração das empresas nas notas 4 e 5 .

Conforme o engenheiro da Empresa F, a resistência a mudanças é a maior dificuldade enfrentada, principalmente durante o processo de implantação. Em geral, a maior resistência 
provém de parte da mão-de-obra e de alguns mestres-de-obras quanto ao uso dos procedimentos padronizados. Segundo o engenheiro, muitas pessoas apresentam resistência em alterar a forma de realizar os serviços ou a tecnologia utilizada.

\subsection{Burocracia excessiva}

Com média 4,14, o excesso de burocracia está em segundo lugar na lista das maiores dificuldades enfrentadas. Além disso, 50\% das empresas afirmam que a burocracia excessiva é a principal dificuldade do programa, conforme a Tabela 1. Ao contrário, do que acontece com o item cultura organizacional, a burocracia excessiva apresenta uma distribuição mais variável. Isso se deve por duas razões. A Empresa B, por exemplo, possui uma pessoa especificamente designada para trabalhar com o programa. Já a Empresa J realmente possui um sistema desburocratizado. Isso se deve à orientação prestada pela consultoria particular contratada pela empresa, que abandonou a consultoria prestada pelo SENAI. As instruções de serviço foram escritas juntamente com os funcionários, de maneira bem simples, para que haja compreensão e sejam utilizados sem resistência. A empresa possui apenas cinco processos principais, relacionados às macros atividades. As demais empresas, que seguiram com a consultoria do SENAI, possuem um número bem maior de processos.

Para a Empresa A, o sistema gera muita burocracia para uma empresa de pequeno porte. $\mathrm{O}$ engenheiro afirma que não há necessidade de fazer listas para si mesmo. Já para a Empresa L, o programa faz muitas exigências de documentação sem necessidade. Um exemplo é a realização de calibração periódica de equipamentos.

Os funcionários operacionais apresentam dificuldades em trabalhar com documentação, principalmente as fichas de verificação de serviços - FIS. Em diversas empresas há a reclamação de que o preenchimento dessas fichas representa perda de tempo sem ganho justificado, que poderia ser mais bem utilizado em outras atividades.

\subsection{Baixo nível de escolaridade dos funcionários}

O baixo nível de escolaridade dos funcionários apresenta a terceira maior média. Entretanto, não há consenso entre os entrevistados devido a grande variabilidade das respostas, como se observa na Tabela 1. Ou seja, para algumas empresas o baixo nível de escolaridade representa um problema, enquanto que para outras não. Segundo alguns engenheiros, o que importa é que o funcionário saiba desempenhar bem suas funções, de modo que um treinamento adequado é o suficiente para obter um serviço de qualidade.

De acordo com a Empresa E, seria mais fácil para os funcionários compreenderem o programa se tivessem maiores níveis de escolaridade. Uma alternativa para contornar esse problema 
é somente realizar a contratação de funcionários alfabetizados, conforme realizado na Empresa D. Já para a Empresa G, a baixa escolaridade dos funcionários não representa problema, uma vez que os conhecimentos necessários são transmitidos através de treinamentos. O engenheiro responsável pelos treinamentos possui habilidade para lidar com pessoas e explica tudo na linguagem utilizadas pelos funcionários.

\subsection{Falta de treinamento}

O quarto item com maior média é a falta de treinamento. Entretanto, as opiniões são muito variáveis, como se pode observar na Tabela 1. Deste modo, algumas empresas vivenciam dificuldades devido à falta de treinamento da mão-de-obra, enquanto que para outras, o treinamento admissional oferecido no canteiro é suficiente para sanar as deficiências.

Salienta-se aqui a deficiência em treinamento tanto em questões relativas à qualidade como em treinamento relacionado às próprias atividades desenvolvidas em canteiro de obra, pelas quais os funcionários são contratados para exercer. O setor da construção civil apresenta grande necessidade de treinamento devido à baixa qualificação de seus funcionários que, aprendem a profissão na prática e pela observação.

\subsection{Falta de envolvimento dos funcionários}

A falta de envolvimento dos funcionários é a quarta maior dificuldade enfrentada, com média 2,57. Deve-se em parte ao desconhecimento do programa e de seus benefícios, bem como de uma certa resistência ao novo. A Empresa C, por exemplo, relata falta de envolvimento dos funcionários no início da implantação, quando muitos funcionários não queriam contribuir.

Entretanto, a empresa afirma que os funcionários colaboram quando percebem as vantagens de trabalhar com melhor qualidade, de acordo com os procedimentos. Para isso, é necessário mostrar a maneira mais eficiente de realizar os serviços e explicar as conseqüências negativas dos erros, tanto para a empresa como para o funcionário. Desta forma, há maior valorização e integração dos trabalhadores, que entendem que fazem parte do mesmo negócio.

\subsection{Falta de participação e conscientização dos colaboradores}

Algumas empresas enfrentam dificuldades com relação aos fornecedores de materiais. A Empresa $\mathrm{C}$ afirma que muitos fornecedores não entregam o material conforme especificado. Deste modo, a empresa é obrigada a realizar inspeção em $100 \%$ do material entregue por novos 
fornecedores. $\mathrm{O}$ número de inspeções diminui com a passar do tempo, a medida em que o fornecedor torna-se mais confiável em suas entregas.

A Empresa $\mathrm{H}$ também enfrenta algumas dificuldades com relação aos fornecedores de materiais e com alguns serviços terceirizados, como a marcação da obra, quando a empreiteira não possui os equipamentos necessários devidamente calibrados. Além disso, a empresa relata uma certa resistência por parte de alguns projetistas terceirizados quanto à adequação aos padrões da empresa. Isso acarreta dificuldades de compatibilização de projetos, o que pode levar a erros durante a execução da obra. Além da forma de apresentação, a empresa também sofre com atrasos em relação ao prazo de entrega dos projetos terceirizados.

\subsection{Comunicação deficiente}

Em geral, os problemas de comunicação deficiente ocorrem entre escritório e obra e entre departamentos da empresa. Na Empresa $\mathrm{C}$, algumas pessoas detentoras de informação não queriam compartilha-las com os demais. Os procedimentos escritos tiveram importante função na difusão do conhecimento na empresa.

Um dos principais problemas na Empresa I diz respeito à comunicação e integração no escritório. Há problemas de relacionamento entre os departamentos de compras e de engenharia. As freqüentes confusões com os pedidos de materiais culminou com a substituição do gerente de compras. Também há certa dificuldade de comunicação entre a direção e demais setores da empresa.

\subsection{Ansiedade por resultados}

Em algumas empresas houve uma certa ansiedade por resultados, como nas Empresas I e G, devido à alta cobrança por parte da direção por resultados. Tal fato ocorreu em menor intensidade em outras empresas. Uma empresa de consultoria foi contratada pela Empresa C para orienta-la durante o processo de certificação. Assim, não houve ansiedade por resultados, devido ao esclarecimento fornecido pela consultoria a respeito do tempo necessário para que surjam os benefícios. Todo o sistema foi implementado no escritório para posteriormente ser levado à obra. Já na Empresa M, houve certa frustração após a certificação, quando se verificou que algumas coisas continuavam a ser executadas da maneira antiga.

\subsection{Falta de comprometimento da alta administração}


Em algumas empresas, a alta administração está totalmente comprometida com o programa e participa ativamente de sua implantação. Já em outras empresas observa-se menor engajamento. Em tais empresas, a diretoria fornece os recursos necessários para a obtenção do certificado, mas se a CEF não exigisse o certificado, a direção iria abolir o programa.

Inicialmente, a Empresa $\mathrm{J}$ sofreu grande resistência da alta administração quanto à implantação do PBQP-H. Alguns engenheiros também não acreditavam no programa. Esta visão negativa começou a mudar com a observação de alguns benefícios, mas ainda não estão plenamente engajados com o sistema. Já na Empresa E, o presidente da empresa não considera a certificação importante, embora os demais engenheiros apóiem a iniciativa.

A Empresa B, por exemplo, afirma que falta colaboração de todos. A direção decidiu pela implantação e contratou uma pessoa especificamente para se responsabilizar pelo programa. Assim, tudo o que diz respeito ao programa é de responsabilidade da assessora da qualidade, de modo que a direção e os engenheiros não se envolvem muito com o sistema. Na Empresa G, a diretoria apóia o programa por meio do fornecimento de recursos e metas, mas poderia participar mais. Segundo o engenheiro, seria melhor se a alta administração comparecesse mais em reuniões, principalmente com os níveis mais baixos, de modo a promover maior envolvimento e demonstrar que a qualidade tem prioridade na empresa.

\subsection{Demais elementos}

A falta de recursos está empatada com a falta de liderança e com a falta de comprometimento dos gerentes, com média 1,93. Em geral, as empresas dispõem dos recursos necessários, principalmente para a realização das auditorias e contratação de pessoal. A falta de liderança também parece não ser um problema para as empresas entrevistadas. Em geral, os engenheiros reconhecem a importância do papel do líder no desenvolvimento dos funcionários para a implantação de um sistema de gestão da qualidade. Entretanto, parece não haver um pleno entendimento por parte dos engenheiros a respeito do conceito de liderança.

Em geral, o nível médio é o mais comprometido com o programa. Composto por engenheiros, técnicos e mestres-de-obras contratados pelas empresas, são essas pessoas que, na maioria das vezes, são encarregadas pela implantação e manutenção do sistema de gestão da qualidade. Mesmo assim, alguns engenheiros têm uma tendência a centralizar informações, de modo que dificilmente delegam tarefa. Finalmente, apenas duas empresas admitem que a falta de foco no cliente pode ter atrapalhado o desenvolvimento da implantação do sistema de gestão da qualidade. A grande maioria (79\%) atribuiu nota mínima para este item, conforme a Tabela 1. 


\section{Conclusões}

Em todo o mundo realizam-se pesquisas sobre a implantação de sistemas de gestão da qualidade e as respectivas dificuldades enfrentadas no processo. Alguns elementos apontados por tais pesquisas são a falta de comprometimento da alta administração, bem como do nível gerencial e operacional, além da resistência a mudanças e forte cultura organizacional. A falta de liderança e de recursos para a realização de melhorias também são citadas como grandes empecilhos ao sucesso das iniciativas voltadas à gestão da qualidade. Outros fatores apontados são as deficiências na comunicação e o excesso de burocracia gerada pelo sistema.

A presente pesquisa, realizada com 14 construtoras da Grande Florianópolis, procura demonstrar a realidade vivida por essas empresas ao implantar e certificar seus sistemas de gestão da qualidade. Em comparação com outras pesquisas, a falta de comprometimento não representa uma dificuldade muito significativa para este grupo de empresas. Em contraste, o item cultura organizacional e resistência a mudanças aparece como a maior média de todos os itens avaliados, o que indica ser este a maior dificuldade enfrentada pelas construtoras.

Deste modo, observa-se que, de maneira geral, todos apóiam o programa, mas apenas até um certo limite. Muitos engenheiros relatam que há grande resistência a mudanças por parte de todos, principalmente devido à cultura conservadora existente na construção civil. Grande parte dessa resistência deve-se à total falta de hábito da documentação dos processos. Uma vez que a realização de registros é uma exigência para a obtenção do certificado, o sistema trouxe uma maior carga burocrática para as empresas. Observa-se uma certa relutância quanto ao preenchimento de fichas e documentos, independente do nível hierárquico. Entretanto, é no nível operacional que se observam as maiores reclamações quanto à burocracia.

Ao analisar os resultados, percebe-se que esses dois itens apresentam as maiores médias, bem superiores aos demais itens. A terceira maior dificuldade refere-se ao baixo nível de escolaridade dos funcionários, seguido pela falta de treinamento e de envolvimento dos funcionários. Todos os demais elementos apresentam pequenas variações nas médias entre um item e outro, pois foram enfrentados em intensidades diferentes pelas empresas.

Assim, observa-se que há muito a fazer em termos de melhoria da qualidade nas empresas construtoras. Além de colaborar para uma melhor compreensão da evolução da qualidade na construção civil, este artigo procura salientar aspectos críticos para a implantação de sistemas de 
gestão da qualidade, o que pode auxiliar outras empresas que ainda não completaram ou não iniciaram o processo de certificação.

\begin{abstract}
The number of construction companies who search for a quality management system certificate has grown in recent years, based mainly in ISO 9000 and PBQP-H. However, the companies pass for a series of difficulties during the process of implementation and certification. The present work has the objective of identify the companies' perceptions about theses difficulties. The research was made with 14 construction companies in Florianópolis city, certified on level A of PBQP-H. This research is conducted with structured interviews, based on a form, with the quality responsible of each company, who evaluate the importance level of a set of items presented with a scale of five points (Likert). As a result, the main difficulties are the cultural aspects and the resistance to changes, beyond the excess of bureaucracy generated for the system. Beyond collaborating for one better understanding of the evolution of the quality in the civil construction, this article pointed critical aspects for the implementation of quality management systems, what it can assist other companies that had not yet completed or initiated the certification process.
\end{abstract}

Key-words: quality; PBQP-H; difficulties.

\title{
Referências
}

ANTONY, Jiju; LEUNG, Kevin; KNOWLES, Graeme; GOSH, Sid. Critical success factors of TQM implementation in Hong Kong industries. International Journal of Quality and Reliability Management, v. 19, n. 5, p. 551-566, 2002. cross ${ }^{\text {ref }}$

BAUER, Pablo; BRANDLI, Luciana L. As dificuldades encontradas por empresas construtoras no processo de certificação do PBQP-H. In: SIMPÓSIO BRASILEIRO DE GESTÃO E ECONOMIA DA CONSTRUÇÃO, IV, 2005, Porto Alegre. Anais... Porto Alegre-RS, 2005. 10 p.

DALGLEISH, Scott. The wrong road toward improvement. Quality, v. 43, n. 13, p. 14, 2004.

LEE, Tat Y. The development of ISO 9000 certification and the future of quality management: a survey of certified firms in Hong Kong. International Journal of Quality and Reliability Management, v. 15, n. 2, p. 162-177, 1998. cross ${ }^{\text {ref }}$

LIU, Chun Kit. Pitfalls of total quality management in Hong Kong. Total Quality Management, v. 9, n. 7, p. 585-598, 1998.

crossef

LOW, Sui Pheng; OMAR, Hennie Faizathy. The effective maintenance of quality management systems in the construction industry. International Journal of Quality and Reliability Management, v. 14, n. 8, p. 768-790, 1997.

LOW, Sui Pheng; TEO, Jasmine Ann. Implementing total quality management in construction firms. Journal of Management in Engineering, v. 20, n. 1, p. 8-15, 2004.

crossef

McINTYRE, Charles; KIRSCHENMAN, Merlin. Survey of TQM in construction industry in uppermidwest. Journal of Management in Engineering, v. 16, n. 5, p. 67-70, 2000.

MENDES, Alexandre Vasconcelos Tajra; PICCHI, Flávio Augusto. Avaliação de implantação de sistemas evolutivos de gestão da qualidade: estudo exploratório em construtoras do estado do Piauí. In: SIMPÓSIO BRASILEIRO DE GESTÃo E ECONOMIA DA CONSTRUÇÃO, IV, 2005, Porto Alegre. Anais... Porto Alegre-RS, 2005,10 p. 
NEVES, C.M.M. Alguns aspectos que interferem na implantação de melhorias e inovações tecnológicas na construção de edifícios. In: ENCONTRO NACIONAL DE TECNOLOGIA DO AMBIENTE CONSTRUÍDO, Rio de Janeiro, 1995, Anais..., Rio de Janeiro: UFRJ/ANTAC, 1995, p.79-84.

PRADO, Renato Lucio; AMARAL, Tatiana Gondim do; TOLEDO, Raquel de. Diretrizes e resultados da implantação do programa $5 S$ na construção civil. In: SIMPÓSIO BRASILEIRO DE GESTÃO DA QUALIDADE E ORGANIZAÇÃO DO TRABALHO NO AMBIENTE CONSTRUÍDO, II, 2001, Fortaleza, Anais... Fortaleza-CE, 2001, 16p.

REIS, Palmyra F.; MELHADO, Silvio B. Implantação de sistemas de gestão da qualidade em empresas de construção de edifícios. In: ENCONTRO NACIONAL DE TECNOLOGIA DO AMBIENTE CONSTRUÍDO, VII, 1998, Florianópolis. Anais... Florianópolis-SC, 1998. p. 619-626.

SILA, I.; EBRAHIMPOUR, M. Examination and comparison of the critical factors of total quality management (TQM) across countries. International Journal of Production Research, v. 41, n. 2, p. 235-268, 2003.

\section{cross ${ }^{\text {ref }}$}

SILVEIRA, Débora Rocha Dias da; AZEVEDO, Eline Silva de; SOUZA, Dayse da Mata Oliveira de; GOUVINHAS, Reidson Pereira. Qualidade na construção civil: um estudo de caso em uma empresa da construção civil no Rio Grande do Norte. In: ENCONTRO NACIONAL DE ENGENHARIA DE PRODUÇÃO, XXII, 2002, Curitiba. Anais... Curitiba-PR, 2002. 8 p.

SOUZA, Roberto de; MEKBEKIAN, Geraldo. Entraves comportamentais e de gestão na implantação de sistemas da qualidade em empresas construtoras. In: ENCONTRO NACIONAL DE TECNOLOGIA DO AMBIENTE CONSTRUÍDO, IV, 1995, Rio de Janeiro. Anais... Rio de Janeiro-RJ, 1995. p.237-242.

SUTHERLAND, Valerie; MAKIN, Peter; BRIGHT, Kevin; COX, Charles. Quality behaviour for quality organizations. Leadership \& Organization Development Journal, v. 16, n. 6, p. 10-15, 1995.

TATIKONDA, Lakshmi U.; TATIKONDA, Rao J. Top ten reasons your TQM effort is failing to improve profit. Production and Inventory Management Journal, v. 37, n. 3, p. 5-9, 1996.

TAYLOR, W.A.; WRIGHT, G.H. The impact of senior managers' commitment on the success of TQM programmes: an empirical study. International Journal of Manpower, v. 24, n. 5, p. 535-550, 2003.

TOLOVI JR., José. Por que os programas de qualidade falham? Revista de Administração de Empresas, v. 34, n. 6, p. 6-11, nov/dez 1994.

TURK, A.M. ISO 9000 in construction: an examination of its application in Turkey. Building and Environment, v. 41, n. 4, p. 501-511, 2006.

cross

\section{Dados dos autores:}

Nome completo: Marcelo Dalcul Depexe, M.Sc.

Filiação institucional: Universidade Federal de Santa Catarina - UFSC

Departamento: Programa de Pós-Graduação em Engenharia de Produção - PPGEP

Endereço completo para correspondência: Rua Conselheiro Laurindo, 557, ap 306. Curitiba/PR Brasil. CEP 80060-100

Telefones para contato: (41) 3330-7218

e-mail: marcelodepexe@yahoo.com.br

Nome completo: Edson Pacheco Paladini, Dr. 
Filiação institucional: Universidade Federal de Santa Catarina - UFSC

Departamento: Programa de Pós-Graduação em Engenharia de Produção - PPGEP

Função ou cargo ocupado: Professor Titular

Endereço completo para correspondência: CTC/UFSC Cx. postal 476. Florianópolis/SC - Brasil. CEP 88010-970

e-mail: paladini@deps.ufsc.br

Recebido para publicação em: 28/10/2006

Aceito para publicação em: 27/02/2007 